\title{
All-optical inverter based on carbon nanotube-polyvinyl alcohol thin film
}

\author{
Zhengpeng Shao (邵正朋), Kan Wu (吴 㑆)*, and Jianping Chen (陈建平) \\ Shanghai Institute for Advanced Communication and Data Science, \\ State Key Laboratory of Advanced Optical Communication Systems and Networks, \\ Department of Electronic Engineering, Shanghai Jiao Tong University, Shanghai 200240, China \\ *Corresponding author: kanwu@sjtu.edu.cn \\ Received December 26, 2019; accepted March 6, 2020; posted online May 13, 2020
}

\begin{abstract}
All-optical light control plays an important role in optical signal processing and communications. In this Letter, we demonstrate an all-optical inverter using carbon nanotube (CNT)-polyvinyl alcohol (PVA) thin film and obtain a long-time stable output due to the environmental insensitivity of the device. The thermo-optic effect in the CNT-PVA thin film generates a thermal lens and modifies the beam propagation in the thin film. The obtained all-optical inverter has a front (trailing) time constant of $\sim 55 \mu \mathrm{s}(44 \mu \mathrm{s})$ for $1550 \mathrm{~nm}$ signal pulses and $\sim 7 \mathrm{kHz}$ response bandwidth.

Keywords: all-optical inverter; low dimensional material; thermo-optic effect. doi: 10.3788/COL202018.060603.
\end{abstract}

Low dimensional materials (LDMs) have attracted wide attention due to their plentiful photonic and optoelectronic properties. Various LDMs have been reported, including graphene ${ }^{[1-5]}$, graphene oxide $\stackrel{[6,7]}{-[}$, carbon nanotubes $(\mathrm{CNTs}) \stackrel{[8-10]}{-1}$, transition metal dichalcogenides (TMDs) $\underline{[1,12]}$, topological insulators (TIs) $\stackrel{[13-15]}{-1}$ black phosphorus (BP) $\stackrel{[16,17]}{ }$, MXene $e^{[18,19]}$, etc. Many optical devices based on these LDMs have been demonstrated such as optical modulators $-\underline{[2,11,20]}$, photodetectors $\stackrel{[4,17]}{-1}$ polarizers ${ }^{[21]}$, logic gates ${ }^{[22]}$, and switches $\stackrel{[12,23]}{-2}$. Among these LDMs, CNTs have attracted research interest since their first early demonstration as saturable absorbers for mode-locked fiber lasers ${ }^{[9,-10]}$. Different from many two-dimensional materials, CNTs have a tunable absorption band and can generate strong absorption as well as a large modulation depth for mode-locking operation.

Meanwhile, all-optical light control plays an important role in optical signal processing and communications because it can effectively avoid the conversions between optical signals and electrical signals and thus can fully utilize the optical bandwidth capacity as well as the manipulation of dimensions including amplitude, phase, and polarization $\stackrel{[23-25]}{-2}$. In recent years, there have been some demonstrations of all-optical modulators and phase shifters based on LDMs. In 2015, Gan et al. first demonstrated an all-fiber phase shifter and switch with a Mach-Zehnder interferometer (MZI) structure based on the photothermal effect of graphene-coated microfiber ${ }^{[26]}$. In 2016, Gan and Wang demonstrated the all-optical switch based on graphene's photothermal effect using a microfiber resonator and a Bragg grating, respectively ${ }^{[27,28]}$. In 2017, Wu et al. demonstrated an all-optical phase shifter and switch with an MZI structure near $1550 \mathrm{~nm}$ using tungsten disulfide $\left(\mathrm{WS}_{2}\right)$ deposited on the tapered fiber ${ }^{[12]}$. In 2018, Wang et al. demonstrated an all-optical modulator with a polarization interference (PI) structure based on molybdenum disulfide $\left(\mathrm{MoS}_{2}\right)$ thin film, which has a more stable output signal under environmental disturbances ${ }^{[11]}$. In these works and many others, the thermo-optic effect is utilized as a phase shifter to modify the phase of the signal light propagating through the materials. However, the thermo-optic effect can also induce transmission reduction, which has not been explored yet.

In this Letter, we demonstrate an all-optical inverter using CNT-polyvinyl alcohol (PVA) thin film. The thermooptic effect in CNT-PVA thin film generates a thermal lens and modifies the beam propagation in the thin film. By embedding the CNT-PVA thin film between two fiber connectors, this thermal lens increases the coupling loss when the light propagates from the CNT-PVA film to the fiber connector. The generation and elimination of a thermal lens in CNT-PVA thin film can be controlled by applying a control light (pump). With this design, an all-optical inverter can be made with respect to the control light. The demonstrated device has time constants of $55 \mu \mathrm{s}$ for the front edge and $44 \mu \mathrm{s}$ for the trailing edge, which indicates the fast thermo-optic response of CNTPVA thin film. Moreover, since the control light and signal light propagate in the same fiber, the all-optical inverter is less sensitive to environmental perturbations. This work proves the potential of LDM CNT in all-optical signal processing systems.

A high-quality CNT-PVA thin film is the key component of the all-optical inverter. The PVA material has a relatively high thermo-optic effect and is easy to mix with CNTs to form a high-quality thin film. There are many other works that used CNT-PVA thin film and show the good performance of this combination ${ }^{[29-31]}$. The LDM CNTs have a strong third-order optical nonlinearity and an ultra-short recovery time of $<1$ ps measured by the pump-probe method ${ }^{[32,33]}$. By using the catalytic vapor decomposition method, single-wall CNTs with $1 \mathrm{~nm}$ to 
$1.5 \mathrm{~nm}$ diameters are synthesized. Then the CNTs are mixed with PVA to form thin films with the following steps. The filiform CNTs are dispersed in the deionized water for $5 \mathrm{~h}$ by an ultrasonic cleaner to obtain $0.5 \mathrm{mg} / \mathrm{mL}$ CNTs dispersion. The CNTs dispersion is then centrifuged at $12,000 \mathrm{~g}$ for several hours. The upper supernatant is collected to reduce the unwanted scattering loss. Then the CNTs dispersion is mixed with a $10 \%$ (mass fraction) aqueous PVA solution at a 2:1 volume ratio for $3 \mathrm{~h}$ by using a magnetic stirrer. Finally, the CNT-PVA mixture is transferred to a clean plastic dish for two-day evaporation to form a thin film. The transmission electron microscope (TEM) image of the CNTs is shown in Fig. 1(a). The thickness of CNT-PVA thin film is $20-30 \mu \mathrm{m}$ measured by a scanning electron microscope (SEM), as shown in Fig. 1(b). Figure 1(c) shows the image of fabricated CNT-PVA thin film transferred on an angled fiber connector. Then the CNT-PVA thin film is sandwiched between two angled fiber connectors (FC/APC) for the experimental usage. The transmission of the CNT-PVA thin film is also measured, as shown in Fig. 1(d). From Fig. 1(d), it can be seen that the transmittance of the CNT-PVA thin film is $43 \%$ at $980 \mathrm{~nm}$, corresponding to a $3.7 \mathrm{~dB}$ insertion loss, and $47 \%$ at $1550 \mathrm{~nm}$, corresponding to a $3.3 \mathrm{~dB}$ insertion loss. The film reaches its melting point of $\sim 80^{\circ} \mathrm{C}$ when $60 \mathrm{~mW}$ power is applied through a fiber, corresponding to a temperature increase of $57^{\circ} \mathrm{C}$ (assuming room temperature $23^{\circ} \mathrm{C}$ ). So the photo-thermal conversion efficiency of CNT-PVA thin film is estimated to be $57^{\circ} \mathrm{C} / 60 \mathrm{~mW}=0.95^{\circ} \mathrm{C} / \mathrm{mW}$.

Before the experiment of the all-optical inverter, we first investigate the relationship between the insertion loss of the CNT-PVA thin film and the incident optical power of the $980 \mathrm{~nm}$ control light (pump). As shown in Fig. 2(a), a fiber MZI is implemented, which mainly consists of a CNT-PVA thin film sandwiched between two angled fiber
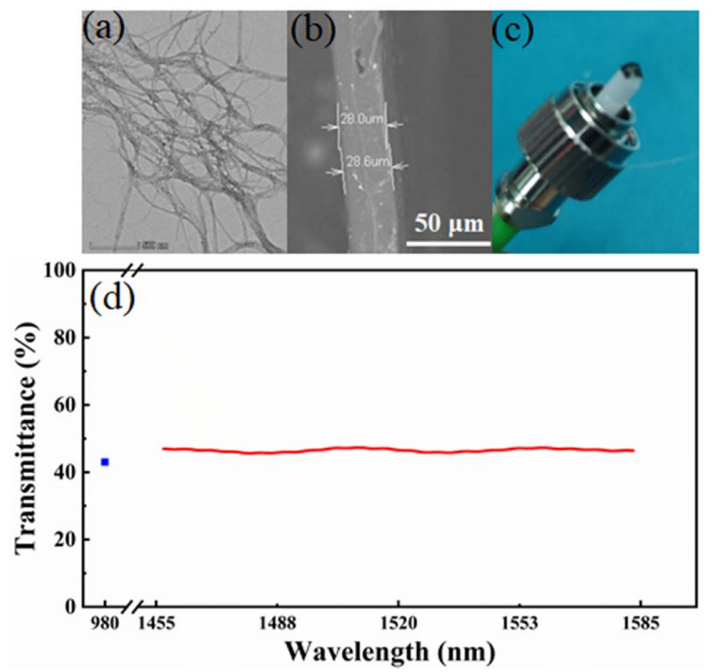

Fig. 1. (a) TEM image of the CNTs in the dispersions. (b) The SEM image of the CNT-PVA thin film. (c) The CNT-PVA thin film transferred onto a fiber connector. (d) The transmittance of the CNT-PVA thin film.
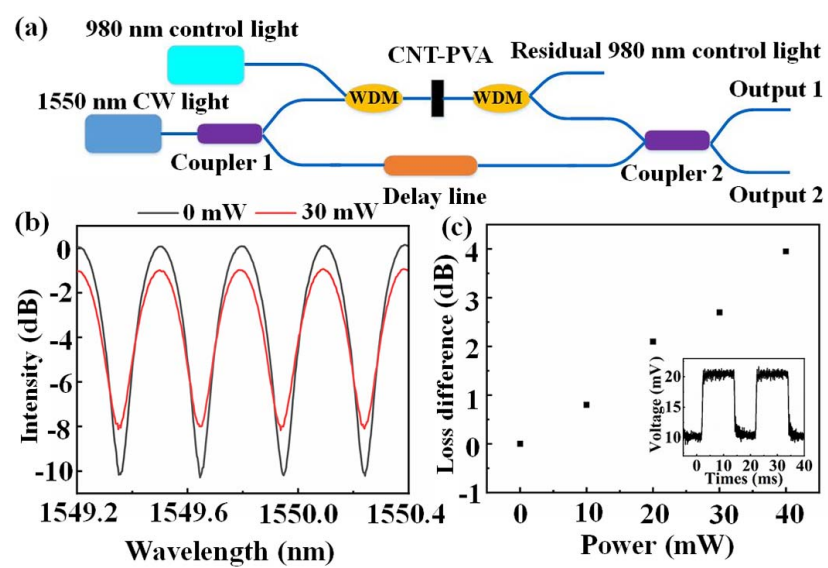

Fig. 2. (a) Experimental setup to measure the power-dependent loss of the CNT-PVA thin film. (b) The transmission spectra of the MZI at the pump power of $0 \mathrm{~mW}$ (black) and $30 \mathrm{~mW}$ (red). (c) The change of insertion loss of the CNT-PVA thin film with the increase of pump power (control light) at $980 \mathrm{~nm}$. Inset: the modulated $1550 \mathrm{~nm}$ output light at the $30 \mathrm{~mW} 980 \mathrm{~nm}$ control light (pump).

connectors, a $980 \mathrm{~nm}$ control light (pump), a $1550 \mathrm{~nm}$ continuous wave (CW) signal light, two wavelength division multiplexers (WDMs), and a delay line. When the $1550 \mathrm{~nm}$ signal light is applied, an interference spectrum can be observed on the optical spectrum analyzer due to the unequal arm length in the MZI, as shown in Fig. 2(b). The period in the spectrum is dependent on the delay between the two arms of the MZI. The contrast between the highest transmission and lowest transmission is dependent on the loss difference between the two arms.

When the $980 \mathrm{~nm}$ control light is injected through a WDM, the CNT-PVA thin film absorbs the power and forms a thermal lens due to the thermo-optic effect. A simulation is performed to explain the mechanism of the thermal lens in our device. The simulation result of beam propagation in the CNT-PVA thin film is shown in Fig. 3(a). The thickness of the thin film is $25 \mu \mathrm{m}$. The initial beam width is $9.5 \mu \mathrm{m}$ from a single-mode fiber, corresponding to the position of Length $=0$ in Fig. 3(a). The temperature gradient is assumed to be $50^{\circ} \mathrm{C}$, that is, the temperature at the position of Length $=25 \mu \mathrm{m}$ is $50^{\circ} \mathrm{C}$ higher than that at Length $=0$. Due to the negative thermo-optic effect of $\mathrm{PVA}\left(-10^{-4} /{ }^{\circ} \mathrm{C}\right)$, an inverse
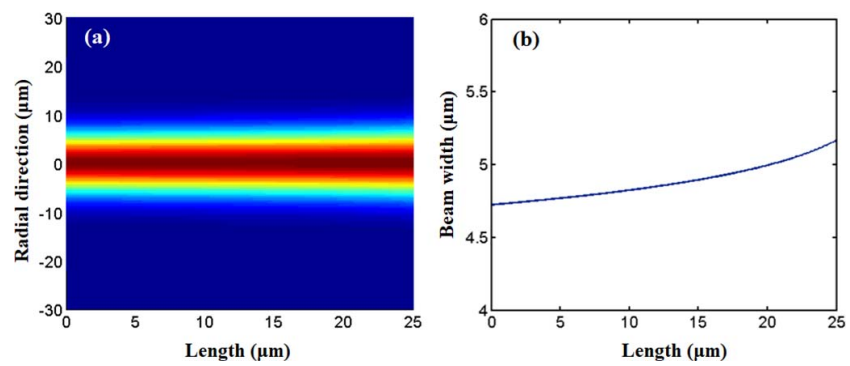

Fig. 3. (a) Simulation of the beam propagation in the heated CNT-PVA thin film. (b) The evolution of beam width. 
thermal lens is formed along the beam propagation direction, that is, the beam width is expanded during the propagation. As a result, the coupling efficiency is reduced when the beam enters the second fiber at Length $=25 \mu \mathrm{m}$. Figure $3(\mathrm{~b})$ shows the evolution of the beam width during the propagation. It can be clearly observed that the beam diameter is increased from $9.5 \mu \mathrm{m}$ to $\sim 10.4 \mu \mathrm{m}$.

Due to the reduced coupling efficiency induced by the thermal lens, the insertion loss of the CNT-PVA thin film is increased and the contrast in the transmission spectrum of the MZI is reduced. As shown in Fig. 2(b), it can be clearly observed that the contrast decreases with the increase of the injected $980 \mathrm{~nm}$ light power from $0 \mathrm{~mW}$ to $40 \mathrm{~mW}$. It is known that the relation between the spectral contrast of an MZI and the loss difference in the two $\operatorname{arms} \stackrel{[34]}{ }$ is given by

$$
\mathrm{ER}=\frac{(1+b)^{2}}{(1-b)^{2}}
$$

and

$$
\mathrm{LD}=10 \log \left(\frac{1}{b^{2}}\right)
$$

where ER is the extinction ratio of interference spectrum, $b$ is the amplitude difference between the two arms, and $\mathrm{LD}$ is the power loss difference between the two arms. Therefore, the insertion loss change of the CNT-PVA thin film can be calculated based on the extinction ratio evolution, as shown in Fig. 2(c). It can be observed that as the power of the $980 \mathrm{~nm}$ control light (pump) increases, the insertion loss of the LDM CNT-PVA thin film is increased by $\sim 4 \mathrm{~dB}$. When the power of the $980 \mathrm{~nm}$ control light exceeds $60 \mathrm{~mW}$, the CNT-PVA thin film is damaged by the accumulated power. The inset of Fig. 2(c) shows the modulated $1550 \mathrm{~nm}$ signal light with a $3 \mathrm{~dB}$ extinction ratio when the power of the $980 \mathrm{~nm}$ control light (pump) is $30 \mathrm{~mW}$.

The experimental setup of the all-optical inverter is shown in Fig. 4. A $1550 \mathrm{~nm} \mathrm{CW}$ laser source is used to generate the signal light. The control light (pump) pulse is generated by using the analog modulation port of the $980 \mathrm{~nm}$ light source. The signal light and the control light are combined by a WDM. In order to obtain the best output signal, there are two polarization controllers (PCs) to control the polarization states of the signal light and the

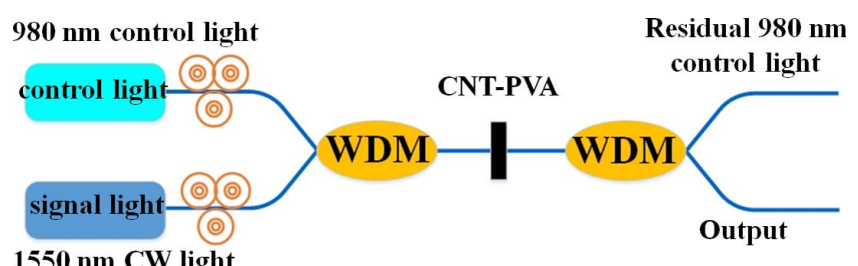

Fig. 4. Experimental setup of an all-optical inverter with the CNT-PVA thin film. control light (pump) before the WDM. The CNT-PVA thin film is sandwiched between two FC/APC fiber connectors. The WDM after the CNT thin film is used to filter out the residual $980 \mathrm{~nm}$ light. When the $980 \mathrm{~nm}$ control light is injected, CNT thin film absorbs the $980 \mathrm{~nm}$ control light (pump), generates heat, forms a thermal lens due to the thermo-optic effect, and changes the power of the $1550 \mathrm{~nm}$ signal light. Then the output signal of the $1550 \mathrm{~nm}$ pulsed light controlled by the $980 \mathrm{~nm}$ control light (pump) can be obtained. The output signal is monitored by a $2.5 \mathrm{GHz}$ oscilloscope (Agilent DSO9254A).

Figure $\underline{5}$ summarizes the property of the output $1550 \mathrm{~nm}$ signal light. We apply the $980 \mathrm{~nm}$ control light with a duty cycle of $20 \%$, a frequency of $50 \mathrm{~Hz}$, and a peak power of $40 \mathrm{~mW}$ into the all-optical inverter system, as shown in Fig. 5(a) (upper panel). Figure 5(a) (lower panel) shows the output waveform of the $1550 \mathrm{~nm}$ signal light. A clear inversion between the output signal and the input control light can be observed. Figure $\underline{5(\mathrm{~b})}$ shows the enlarged view of the single output $1550 \mathrm{~nm}$ pulse. By fitting the front edge (trailing edge) of the output $1550 \mathrm{~nm}$ signal light pulse with the exponential function $\exp \left(-t / t_{f}\right)\left[1-\exp \left(-t / t_{r}\right)\right]$, the time constants of the front and trailing edges are $55 \mu$ s and $44 \mu \mathrm{s}$, respectively. The fitting curves are shown in Fig. $5(\mathrm{~b})$. The operation speed is estimated to be $0.35 / 55 \mu \mathrm{s}=\sim 6.4 \mathrm{kHz}$. In addition, the transition time from $90 \%$ to $10 \%$ ( $10 \%$ to $90 \%$ ) optical power is $137 \mu \mathrm{s}(103 \mu \mathrm{s})$. The time of the front edge of the output waveform (1550 nm signal light) mainly depends on how fast the control light can heat the CNTPVA thin film, while the time of the trailing edge is only determined by the heat dissipation in the CNT-PVA thin film when the control light is absent. The long-term stability of the output signal light is also demonstrated, as

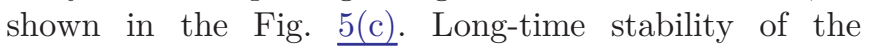

(a)

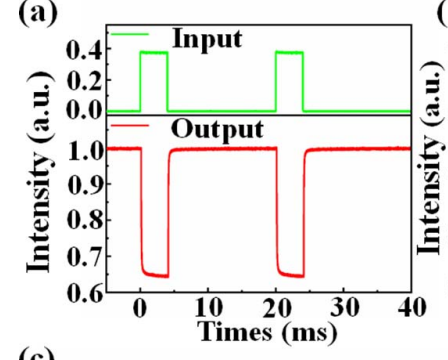

(b)

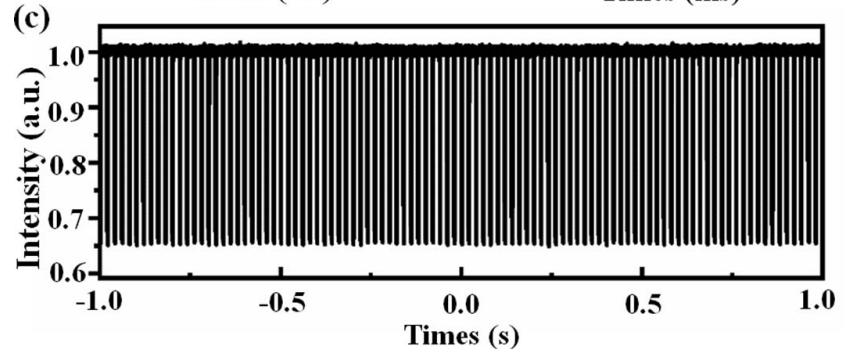

Fig. 5. (a) Pulsed $980 \mathrm{~nm}$ control light (upper) and modulated $1550 \mathrm{~nm}$ output signal (lower). (b) A zoomed view of an output signal (black) and exponential fit (red). (c) A long-term stable output. 
amplitude of the output pulses can be clearly obtained, which proves that our CNT-PVA-based all-optical inverter device is less sensitive to environmental disturbances. The operational wavelength of the all-optical inverter is dependent on the absorption wavelength of the CNTs. As long as the wavelength can be absorbed by the CNTs, it can be used as a pump wavelength. For the signal wavelength, it should have lower absorption in CNTs and PVA.

The time constant of the front (trailing) edge of the output signal light is very important to the all-optical inverter system. We also investigate the relationship between the peak power of $980 \mathrm{~nm}$ control light and the time constant of the front (trailing) edge of the output $1550 \mathrm{~nm}$ signal light. The control light with a different duty cycle and fixed peak power is applied. The output $1550 \mathrm{~nm}$ signal light is shown in Fig. 6(a). The corresponding time constants of the front and trailing edges are summarized in Fig. $\underline{6(\mathrm{c})}$. As discussed above, the time constant of the front edge mainly depends on how fast the control light can heat the CNT-PVA thin film. Therefore, the time constants are nearly unchanged for a fixed peak power of control light. Similarly, the time constant of the trailing edge is only determined by the heat dissipation in the CNT-PVA thin film, which is also nearly unchanged.

When the control light with a different peak power and fixed average power (i.e., fixed pulse energy) is applied, the output signal light is shown in Fig. $\underline{6(\mathrm{~b})}$. The corresponding time constants are summarized in Fig. $\underline{6(d)}$. When the control light has a higher peak power, due to the limited bandwidth of the device (caused by the slow response of the thermal effect), the rising time is increased and the heating process is lengthened. As a result, the time constant for the front edge also increases. Similarly, the time constant of the trailing edge is also increased. When
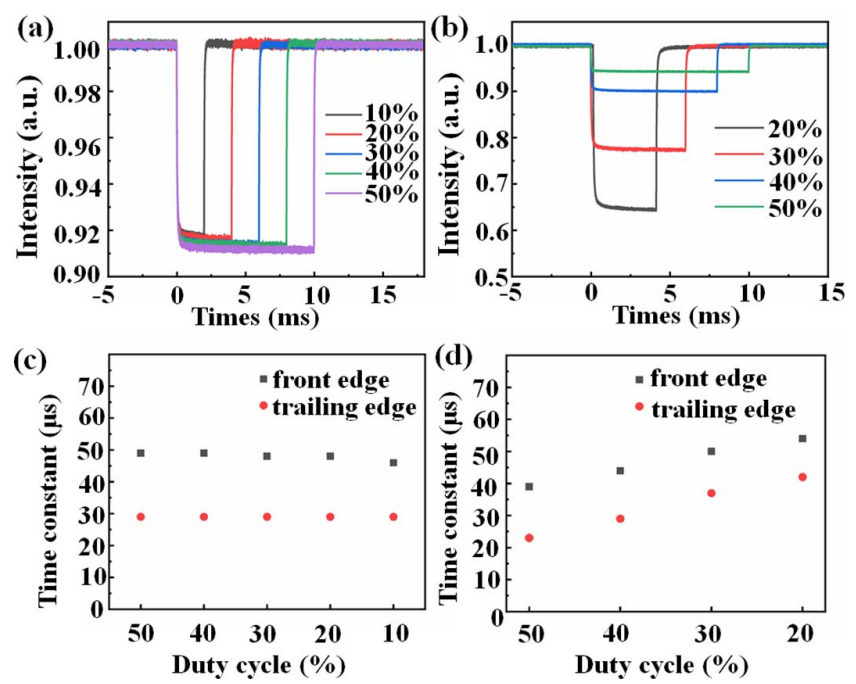

Fig. 6. Output signal pulses and the time constants of the front (trailing) edge when the control light (pump) with different duty cycles and (a), (c) the same peak power or (b), (d) different peak powers are applied. the duty cycle reaches $10 \%$, the corresponding peak power of the control light is $\sim 80 \mathrm{~mW}$, which exceeds the damage threshold of the thin film. Therefore, the experiment with $10 \%$ duty cycle is not performed.

Compared with the all-optical signal processing based on the saturable absorption of low-dimensional materials $(\mathrm{LDMs}) \stackrel{[35-38]}{-3}$, the saturable absorption can provide a very fast response (ps to fs level) and support high-speed operation. However, its modulation depth is typically less than $10 \%$. Therefore, to obtain a better modulation depth, we adopt the thermo-optic effect as our working mechanism. As can be seen, the modulation depth can be $50 \%$.

To verify the thermal accumulation property of the CNT-PVA thin film, a burst of pulses with a period of $2 \mathrm{~ms}$ is applied. Each burst includes three pules at $980 \mathrm{~nm}$ with a frequency of $4 \mathrm{kHz}$ and a duty cycle of $90 \%$. The waveforms of the $980 \mathrm{~nm}$ control light (upper panel) and the output $1550 \mathrm{~nm}$ signal light (lower panel) are shown in Fig. 7. It can be observed that the output $1550 \mathrm{~nm}$ signal light shows the increased negative amplitude in a burst period, which is very similar to the charge-discharge behavior of a capacitance. It is a clear demonstration of the thermal accumulation property of the device.

To further investigate the response bandwidth of the all-optical inverter, the $980 \mathrm{~nm}$ control light (pump) modulated by a sine wave from $50 \mathrm{~Hz}$ to $12 \mathrm{kHz}$ is applied. As shown in Fig. 8(a), when the amplitude of the sine wave is fixed and the frequency of the sine wave gradually increases, the peak-to-peak voltages of the output signal waveforms gradually decrease due to the slow thermooptic effect. In addition, it is obvious in Fig. 8(b) that when the frequency is fixed and the amplitude of the sine wave of the $980 \mathrm{~nm}$ control light (pump) is raised, the peak-to-peak voltage of the output signal also increases. However, the difference is gradually weakened with the increase of the amplitude. This is due to the fact that the CNT-PVA thin film gradually reaches the saturation state. The $3 \mathrm{~dB}$ bandwidth of the all-optical inverter where the amplitude drops to half of the maximum amplitude is $\sim 7 \mathrm{kHz}$.

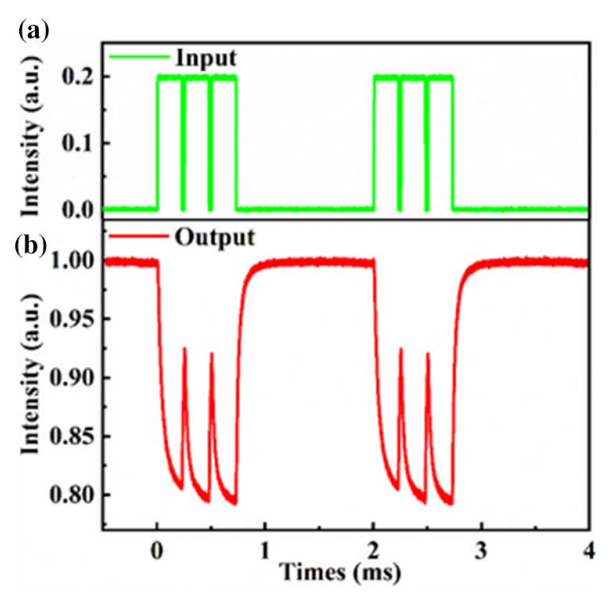

Fig. 7. (a) Burst of the control light (pump). (b) The signal output. 

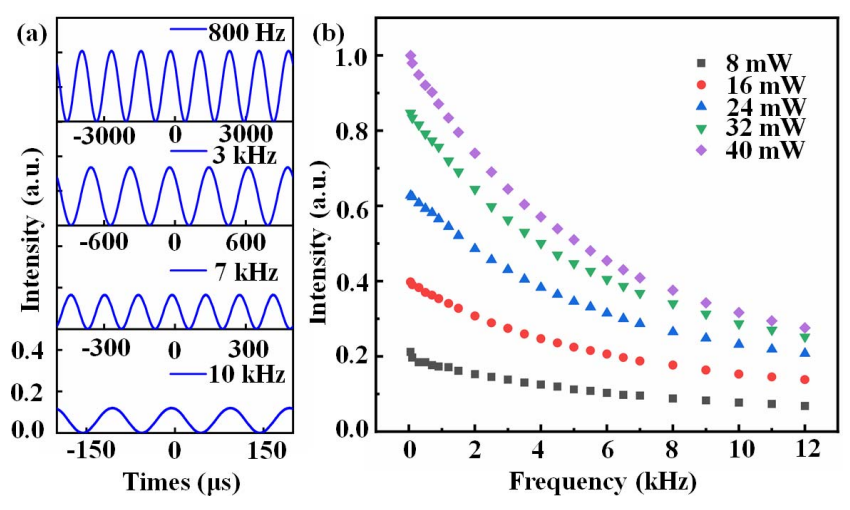

Fig. 8. (a) Output signals with different amplitudes of the $980 \mathrm{~nm}$ control light at different frequencies of the $980 \mathrm{~nm}$ sine wave control light; (b) the corresponding peak-to-peak amplitudes.

In conclusion, we demonstrate an all-optical inverter based on the thermal lens effect in CNT-PVA thin film. It has a front (trailing) time constant of $\sim 55 \mu \mathrm{s}(44 \mu \mathrm{s})$ for $1550 \mathrm{~nm}$ signal pulses. The response bandwidth is $\sim 7 \mathrm{kHz}$. Long-term stability of the output signal is also demonstrated due to the environmental insensitivity of the device. The compactness and high stability of the device indicate the potential of LDM in all-optical communications and signal processing systems.

This work was supported by the National Natural Science Foundation of China (NSFC) (Nos. 61922056 and 61875122).

\section{References}

1. T. Gu, N. Petrone, J. F. McMillan, A. van der Zande, M. Yu, G.-Q. Lo, D.-L. Kwong, J. Hone, and C. W. Wong, Nat. Photonics 6, 554 (2012).

2. M. Liu, X. Yin, E. Ulin-Avila, B. Geng, T. Zentgraf, L. Ju, F. Wang, and X. Zhang, Nature 474, 64 (2011).

3. A. Martinez and Z. Sun, Nat. Photonics 7, 842 (2013).

4. T. Mueller, F. Xia, and P. Avouris, Nat. Photonics 4, 297 (2010).

5. Z. Sun, T. Hasan, F. Torrisi, D. Popa, G. Privitera, F. Wang, F. Bonaccorso, D. M. Basko, and A. C. Ferrari, ACS Nano 4, 803 (2010).

6. D. A. Dikin, S. Stankovich, E. J. Zimney, R. D. Piner, G. H. B. Dommett, G. Evmenenko, S. T. Nguyen, and R. S. Ruoff, Nature 448, 457 (2007).

7. G. Sobon, J. Sotor, J. Jagiello, R. Kozinski, M. Zdrojek, M. Holdynski, P. Paletko, J. Boguslawski, L. Lipinska, and K. M. Abramski, Opt. Express 20, 19463 (2012).

8. R. H. Baughman, A. A. Zakhidov, and W. A. De Heer, Science 297, 787 (2002)

9. S. Y. Set, H. Yaguchi, Y. Tanaka, and M. Jablonski, J. Lightwave Technol. 22, 51 (2004).
10. S. Yamashita, Y. Inoue, S. Maruyama, Y. Murakami, H. Yaguchi, M. Jablonski, and S. Y. Set, Opt. Lett. 29, 1581 (2004).

11. Y. Wang, K. Wu, and J. Chen, Chin. Opt. Lett. 16, 020003 (2018).

12. K. Wu, C. Guo, H. Wang, X. Zhang, J. Wang, and J. Chen, Opt. Express 25, 17639 (2017).

13. J. Lee, J. Koo, Y. M. Jhon, and J. H. Lee, Opt. Express 22, 6165 (2014).

14. J. E. Moore, Nature 464, 194 (2010).

15. H. Zhang, C.-X. Liu, X.-L. Qi, X. Dai, Z. Fang, and S.-C. Zhang, Nat. Phys. 5, 438 (2009).

16. F. Xia, H. Wang, and Y. Jia, Nat. Commun. 5, 4458 (2014).

17. H. Yuan, X. Liu, F. Afshinmanesh, W. Li, G. Xu, J. Sun, B. Lian, A. G. Curto, G. Ye, and Y. Hikita, Nat. Nanotechnol. 10, 707 (2015).

18. X. Jiang, S. Liu, W. Liang, S. Luo, Z. He, Y. Ge, H. Wang, R. Cao, F. Zhang, Q. Wen, J. Li, Q. Bao, D. Fan, and H. Zhang, Laser Photonics Rev. 12, 1700229 (2018).

19. X. Wang, S. Kajiyama, H. Iinuma, E. Hosono, S. Oro, I. Moriguchi, M. Okubo, and A. Yamada, Nat. Commun. 6, 6544 (2015).

20. Z. Sun, A. Martinez, and F. Wang, Nat. Photonics 10, 227 (2016).

21. Q. Bao, H. Zhang, B. Wang, Z. Ni, C. H. Y. X. Lim, Y. Wang, D. Y. Tang, and K. P. Loh, Nat. Photonics 5, 411 (2011).

22. Q. Guo, K. Wu, Z. Shao, E. T. Basore, P. Jiang, and J. Qiu, Adv. Opt. Mater. 1900322 (2019).

23. R. L. Gieseking, S. Mukhopadhyay, C. Risko, S. R. Marder, and J. L. Brédas, Adv. Mater. 26, 68 (2014).

24. D. Richardson, J. Fini, and L. E. Nelson, Nat. Photonics 7, 354 (2013).

25. A. E. Willner, S. Khaleghi, M. R. Chitgarha, and O. F. Yilmaz, J. Lightwave Technol. 32, 660 (2013).

26. X. Gan, C. Zhao, Y. Wang, D. Mao, L. Fang, L. Han, and J. Zhao, Optica 2, 468 (2015).

27. Y. Wang, X. Gan, C. Zhao, L. Fang, D. Mao, Y. Xu, F. Zhang, T. Xi, L. Ren, and J. Zhao, Appl. Phys. Lett. 108, 171905 (2016).

28. X. Gan, Y. Wang, F. Zhang, C. Zhao, B. Jiang, L. Fang, D. Li, H. Wu, Z. Ren, and J. Zhao, Opt. Lett. 41, 603 (2016).

29. Y. Bin, M. Mine, A. Koganemaru, X. Jiang, and M. Matsuo, Polymer 47, 1308 (2006).

30. L. Deng and M.-B. Hägg, Int. J. Greenhouse Gas Control 26, 127 (2014).

31. S. Y. Hu, Y. Zhang, D. Lawless, and X. Feng, J. Membrane Sci. 417, $34(2012)$.

32. Y.-C. Chen, N. R. Raravikar, L. S. Schadler, P. M. Ajayan, Y.-P. Zhao, T.-M. Lu, G.-C. Wang, and X.-C. Zhang, Appl. Phys. Lett. 81, 975 (2002).

33. S. Tatsuura, M. Furuki, Y. Sato, I. Iwasa, M. Tian, and H. Mitsu, Adv. Mater. 15, 534 (2003).

34. G. Agrawal, Applications of Nonlinear Fiber Optics (Elsevier, 2001).

35. Y. Song, Z. Liang, X. Jiang, Y. Chen, Z. Li, L. Lu, Y. Ge, K. Wang, J. Zheng, and S. Lu, 2D Mater. 4, 045010 (2017).

36. Y. Ge, Z. Zhu, Y. Xu, Y. Chen, S. Chen, Z. Liang, Y. Song, Y. Zou, H. Zeng, and S. Xu, Adv. Opt. Mater. 6, 1701166 (2018).

37. M. Zhang, Q. Wu, F. Zhang, L. Chen, X. Jin, Y. Hu, Z. Zheng, and H. Zhang, Adv. Opt. Mater. 7, 1800224 (2019).

38. Y. Song, X. Shi, C. Wu, D. Tang, and H. Zhang, Appl. Phys. Rev. 6, 021313 (2019). 